Commun. Korean Math. Soc. 28 (2013), No. 1, pp. 123-134

http://dx.doi.org/10.4134/CKMS.2013.28.1.123

\title{
ON SEQUENCE OF FUNCTIONS
}

\author{
Ibrahim A. Salehbhai, Jyotindra C. Prajapati, and Ajay K. Shukla
}

\begin{abstract}
Operational techniques have drawn the attention of several researchers in the study of sequence of functions and polynomials. An attempt is made to introduce a new sequence of functions by using operational techniques. Some generating relations and finite summation formulae have been obtained. The corresponding MAPLE code for obtaining above sequence of functions for different values of parameters was also discussed.
\end{abstract}

\section{Introduction}

Operational techniques [1], also known as operational Calculus, have drawn the attention of several researchers in the study of sequence of functions and polynomials. The idea of representing the processes of calculus, derivation, and integration, as operators is called operational technique. By operational technique, the problems in analysis, in particular differential equations are transformed into algebraic problems, usually the problem of solving a polynomial equation.

The key element of the operational technique is to consider differentiation as an operator $D \equiv \frac{d}{d x}$ acting on functions. Linear differential equations can then be recast in the form of an operator valued function $F(D)$ of the operator $D$ acting on the unknown function equals the known function. Solutions are then obtained by making the inverse operator of $F$ act on the known function.

A new sequence of functions $\left\{V_{n}^{(\alpha, \beta)}(x ; a, k, s): n=0,1,2, \ldots\right\}$ is introduced in this paper as:

$$
V_{n}^{(\alpha, \beta)}(x ; a, k, s)=\frac{1}{n !} x^{-\beta} E_{\alpha}\left\{p_{k}(x)\right\}\left(T_{x}^{a, s}\right)^{n}\left[x^{\beta} E_{\alpha}\left\{-p_{k}(x)\right\}\right],
$$

where $T_{x}^{a, s} \equiv x^{a}(s+x D), D \equiv \frac{d}{d x}, a$ and $s$ are constants, $\beta \geq 0, k$ is finite and non-negative integer, $p_{k}(x)$ is a polynomial in $x$ of degree $k, x \in \mathbb{R}$ and $E_{\alpha}(z)$

Received February 9, 2012.

2010 Mathematics Subject Classification. 33E10, 33E12, 33E99, 44A45, 68W30.

Key words and phrases. Mittag-Leffler function, generating relations, finite summation formulae, symbolic representation. 
is the Mittag-Leffler function (M. N. Berberan-Santos [2]) defined as:

$$
E_{\alpha}(z)=\sum_{n=0}^{\infty} \frac{z^{n}}{\Gamma(\alpha n+1)}, \alpha \geq 0 .
$$

Some generating relations and finite summation formulae have been obtained by using the properties of the differential operators $T_{x}^{a, s} \equiv x^{a}(s+x D), T_{x}^{a, 1} \equiv$ $x^{a}(1+x D)$, where $D \equiv \frac{d}{d x}$, based on the work of Mittal [3], Patil and Thakare [4], Srivastava and Singh [7].

Some well-known definitions and facts are listed as below:

Generalized Laguerre polynomials (Srivastava and Manocha [9]) defined as:

$$
L_{n}^{(\alpha)}(x)=\frac{x^{-\alpha-n-1} e^{x}}{n !}\left(x^{2} D\right)^{n}\left[x^{\alpha+1} e^{-x}\right] .
$$

Hermite polynomials (Rainville [6]) defined as:

$$
H_{n}(x)=(-1)^{n} \exp \left(x^{2}\right) D^{n}\left[\exp \left(-x^{2}\right)\right] .
$$

Konhauser polynomials of first kind (Srivastava [8]) defined as:

$$
Y_{n}^{\alpha}(x ; k)=\frac{x^{-k n-\alpha-1} e^{x}}{k^{n} n !}\left(x^{k+1} D\right)^{n}\left[x^{\alpha+1} e^{-x}\right] .
$$

Konhauser polynomials of second kind (Srivastava [8]) defined as: for $k$ is a positive integer,

$$
Z_{n}^{\alpha}(x ; k)=\frac{\Gamma(k n+\alpha+1)}{n !} \sum_{j=0}^{n}(-1)^{j}\left(\begin{array}{c}
n \\
j
\end{array}\right) \frac{x^{k j}}{\Gamma(k j+\alpha+1)} .
$$

$L_{n}^{\alpha, \beta}(x)$ polynomials (Prabhakar and Suman [5]) defined as:

$$
L_{n}^{\alpha, \beta}(x)=\frac{\Gamma(\alpha n+\beta+1)}{n !} \sum_{k=0}^{n} \frac{(-n)_{k} x^{k}}{\Gamma(\alpha k+\beta+1) k !},
$$

$\operatorname{Re} \beta>-1$ and $\alpha$ is any complex number with $\operatorname{Re} \alpha>0$.

The following relations are established by Prabhakar and Suman [5]

$$
\begin{gathered}
L_{n}^{k, \beta}\left(x^{k}\right)=Z_{n}^{\beta}(x ; k), \\
L_{n}^{1, \beta}(x)=L_{n}^{\beta}(x) .
\end{gathered}
$$

Mittal [3] discussed following result:

$$
\exp \left(T_{x}^{a, s}\right)\left[x^{\gamma} f(x)\right]=x^{-\gamma}\left(1-a x^{a} t\right)^{-\left(\frac{\gamma+s}{a}\right)} f\left(x\left(1-a x^{a} t\right)^{-\frac{1}{a}}\right) .
$$

The relationships between sequence of functions (1.1) \& polynomials (1.2)-(1.6) for appropriate values of $\alpha, \beta, a, k$ and $s$ have been obtained. Some cases for $\alpha \neq 1$ discussed with graphs in present paper. 


\section{Generating relations}

The generating relations of (1.1) have been obtained as:

$$
\begin{aligned}
& \sum_{n=0}^{\infty} x^{-a n} V_{n}^{(\alpha, \beta)}(x ; a, k, s) t^{n} \\
= & (1-a t)^{-\left(\frac{\beta+s}{a}\right)} E_{\alpha}\left[p_{k}(x)\right] E_{\alpha}\left[-p_{k}\left\{x(1-a t)^{-\frac{1}{a}}\right\}\right]^{\cdot}\left[-p_{k}\left\{x(1+a t)^{\frac{1}{a}}\right\}\right], \\
& \sum_{n=0}^{\infty} x^{-a n} V_{n}^{(\alpha, \beta-a n)}(x ; a, k, s) t^{n} \\
= & (1+a t)^{\frac{\beta+s}{a}-1} E_{\alpha}\left[p_{k}(x)\right] E_{\alpha}\left[-p_{k}\left\{x(1+a t)^{\frac{1}{a}}\right\}\right], \\
& \sum_{m=0}^{\infty} x^{-a m}\left(\begin{array}{l}
m+n \\
n
\end{array}\right) V_{n+m}^{(\alpha, \beta)}(x ; a, k, s) t^{m} \\
= & (1-a t) \\
& -\left(\frac{\beta+s}{a}\right) \frac{E_{\alpha}\left\{p_{k}(x)\right\}}{E_{\alpha}\left[p_{k}\left\{x(1-a t)^{-\frac{1}{a}}\right]\right.} V_{n}^{(\alpha, \beta)}\left\{x(1-a t)^{-\frac{1}{a}} ; a, k, s\right\} .
\end{aligned}
$$

Proof of (2.1). From (1.1), we consider

$$
\sum_{n=0}^{\infty} V_{n}^{(\alpha, \beta)}(x ; a, k, s) t^{n}=x^{-\beta} E_{\alpha}\left\{p_{k}(x)\right\} \exp \left(t T_{x}^{a, s}\right)\left[x^{\beta} E_{\alpha}\left\{-p_{k}(x)\right\}\right] .
$$

Further simplification gives

$$
\begin{aligned}
& =x^{-\beta} E_{\alpha}\left\{p_{k}(x)\right\} x^{\beta}\left(1-a x^{a} t\right)^{-\left(\frac{\beta+s}{a}\right)} E_{\alpha}\left[-p_{k}\left\{x\left(1-a x^{a} t\right)^{-\frac{1}{a}}\right\}\right] \\
& =\left(1-a x^{a} t\right)^{-\left(\frac{\beta+s}{a}\right)} E_{\alpha}\left\{p_{k}(x)\right\} E_{\alpha}\left[-p_{k}\left\{x\left(1-a x^{a} t\right)^{-\frac{1}{a}}\right\}\right]
\end{aligned}
$$

and replacing $t$ by $t x^{-a}$, this gives (2.1).

Proof of (2.2). Again from (1.1), we have

$$
\begin{aligned}
& \sum_{n=0}^{\infty} x^{-a n} V_{n}^{(\alpha, \beta-a n)}(x ; a, k, s) t^{n} \\
= & x^{-\beta} E_{\alpha}\left\{p_{k}(x)\right\} \exp \left(t T_{x}^{a, s}\right)\left[x^{\beta-a n} E_{\alpha}\left\{-p_{k}(x)\right\}\right],
\end{aligned}
$$

and simplifying the above equation, we get

$$
\begin{aligned}
& \sum_{n=0}^{\infty} x^{-a n} V_{n}^{(\alpha, \beta-a n)}(x ; a, k, s) t^{n} \\
= & x^{-\beta} E_{\alpha}\left\{p_{k}(x)\right\} x^{\beta}(1+a t)^{\frac{\beta+s}{a}-1} E_{\alpha}\left[-p_{k}\left\{x(1+a t)^{\frac{1}{a}}\right\}\right] \\
& \times x^{-\beta} E_{\alpha}\left\{p_{k}(x)\right\} x^{\beta}(1+a t)^{\frac{\beta+s}{a}-1} E_{\alpha}\left[-p_{k}\left\{x(1+a t)^{\frac{1}{a}}\right\}\right] \\
= & (1+a t)^{\frac{\beta+s}{a}-1} E_{\alpha}\left[p_{k}(x)\right] E_{\alpha}\left[-p_{k}\left\{x(1+a t)^{\frac{1}{a}}\right\}\right],
\end{aligned}
$$

this proves $(2.2)$. 
Proof of (2.3). We can write (1.1) as:

$$
\left(T_{x}^{a, s}\right)^{n}\left[x^{\beta} E_{\alpha}\left\{-p_{k}(x)\right\}\right]=n ! x^{\beta} \frac{1}{E_{\alpha}\left\{p_{k}(x)\right\}} V_{n}^{(\alpha, \beta)}(x ; a, k, s)
$$

or

$$
\begin{aligned}
& \exp \left(t T_{x}^{a, s}\right)\left\{\left(T_{x}^{a, s}\right)^{n}\left[x^{\beta} E_{\alpha}\left\{-p_{k}(x)\right\}\right]\right\} \\
= & n ! \exp \left(t T_{x}^{a, s}\right)\left\{x^{\beta} \frac{1}{E_{\alpha}\left\{p_{k}(x)\right\}} V_{n}^{(\alpha, \beta)}(x ; a, k, s)\right\},
\end{aligned}
$$

i.e.,

$$
\begin{aligned}
& \sum_{m=0}^{\infty} \frac{t^{m}\left(T_{x}^{a, s}\right)^{m+n}}{m !}\left[x^{\beta} E_{\alpha}\left\{-p_{k}(x)\right\}\right] \\
= & n ! \exp \left(t T_{x}^{a, s}\right)\left\{x^{\beta} \frac{1}{E_{\alpha}\left\{p_{k}(x)\right\}} V_{n}^{(\alpha, \beta)}(x ; a, k, s)\right\} .
\end{aligned}
$$

This can also be written as:

$$
\begin{aligned}
& \sum_{m=0}^{\infty} \frac{t^{m}\left(T_{x}^{a, s}\right)^{m+n}}{m !}\left[x^{\beta} E_{\alpha}\left\{-p_{k}(x)\right\}\right] \\
= & n ! x^{\beta}\left(1-a x^{a} t\right)^{-\left(\frac{\beta+s}{a}\right)} \frac{1}{E_{\alpha}\left[p_{k}\left\{x\left(1-a x^{a} t\right)^{-\frac{1}{a}}\right\}\right]} \\
& \times V_{n}^{(\alpha, \beta)}\left\{x\left(1-a x^{a} t\right)^{-\frac{1}{a}} ; a, k, s\right\},
\end{aligned}
$$

use of (2.4), yields

$$
\begin{aligned}
& \sum_{m=0}^{\infty} \frac{1}{m ! n !}(m+n) ! x^{\beta} \frac{1}{E_{\alpha}\left\{p_{k}(x)\right\}} V_{m+n}^{(\alpha, \beta)}(x ; a, k, s) t^{m} \\
= & x^{\beta}\left(1-a x^{a} t\right)^{-\left(\frac{\beta+s}{a}\right)} \frac{1}{E_{\alpha}\left[p_{k}\left\{x\left(1-a x^{a} t\right)^{-\frac{1}{a}}\right\}\right]} \\
& \times V_{n}^{(\alpha, \beta)}\left\{x\left(1-a x^{a} t\right)^{-\frac{1}{a}} ; a, k, s\right\} .
\end{aligned}
$$

Therefore,

$$
\begin{gathered}
\sum_{m=0}^{\infty}\left(\begin{array}{l}
m+n \\
n
\end{array}\right) V_{m+n}^{(\alpha, \beta)}(x ; a, k, s) t^{m} \\
=\left(1-a x^{a} t\right)^{-\left(\frac{\beta+s}{a}\right)} \frac{E_{\alpha}\left\{p_{k}(x)\right\}}{E_{\alpha}\left[p_{k}\left\{x\left(1-a x^{a} t\right)^{-\frac{1}{a}}\right\}\right]} \\
\times V_{n}^{(\alpha, \beta)}\left\{x\left(1-a x^{a} t\right)^{-\frac{1}{a}} ; a, k, s\right\},
\end{gathered}
$$

on replacing $t$ by $t x^{-a}$, this leads to (2.3). 


\section{Finite summation formulae}

The finite sum formulae for (1.1) have been obtained as:

$$
\begin{aligned}
& V_{n}^{(\alpha, \beta)}(x ; a, k, s)=\sum_{m=0}^{n} \frac{1}{m !}\left(a x^{a}\right)^{m}\left(\frac{\beta}{a}\right)_{m} V_{n-m}^{(\alpha, 0)}(x ; a, k, s), \\
& V_{n}^{(\alpha, \beta)}(x ; a, k, s)=\sum_{m=0}^{n} \frac{1}{m !}\left(a x^{a}\right)^{m}\left(\frac{\beta-\gamma}{a}\right)_{m} V_{n-m}^{(\alpha, \gamma)}(x ; a, k, s) .
\end{aligned}
$$

Proof of (3.1). We can write (1.1) as

$$
\begin{aligned}
& V_{n}^{(\alpha, \beta)}(x ; a, k, s) \\
= & \frac{1}{n !} x^{-\beta} E_{\alpha}\left\{p_{k}(x)\right\}\left(T_{x}^{a, s}\right)^{n}\left[x x^{\beta-1} E_{\alpha}\left\{-p_{k}(x)\right\}\right], \\
= & \frac{1}{n !} x^{-\beta} E_{\alpha}\left\{p_{k}(x)\right\} x \times \sum_{m=0}^{n}\left(\begin{array}{c}
n \\
m
\end{array}\right)\left(T_{x}^{a, s}\right)^{n-m}\left[E_{\alpha}\left\{-p_{k}(x)\right\}\right]\left(T_{x}^{a, 1}\right)^{m}\left(x^{\beta-1}\right) \\
= & \frac{1}{n !} x^{-\beta+1} E_{\alpha}\left\{p_{k}(x)\right\} \sum_{m=0}^{n} \frac{n !}{m !(n-m) !}\left[E_{\alpha}\left\{-p_{k}(x)\right\}\right] x^{a(n-m)} \\
& \times[(s+x D)(s+a+x D)(s+2 a+x D) \cdots(s+(n-m-1) a+x D)] \\
& \times x^{a m}[(1+x D)(1+a+x D)(1+2 a+x D) \cdots(1+(m-1) a+x D)] x^{\beta-1} \\
= & E_{\alpha}\left\{p_{k}(x)\right\} \sum_{m=0}^{n} \frac{1}{m !(n-m) !} x^{a n} \\
& \quad \times \prod_{n-m-1}(s+i a+x D)\left[E_{\alpha}\left\{-p_{k}(x)\right\}\right] a^{m}\left(\frac{\beta}{a}\right)_{m} .
\end{aligned}
$$

Putting $\beta=0$ and replacing $n$ by $n-m$ in (1.1), we get

$$
V_{n-m}^{(\alpha, 0)}(x ; a, k, s)=\frac{1}{(n-m) !} E_{\alpha}\left\{p_{k}(x)\right\}\left(T_{x}^{a, s}\right)^{n-m}\left[E_{\alpha}\left\{-p_{k}(x)\right\}\right],
$$

i.e.,

$$
\frac{1}{(n-m) !}\left(T_{x}^{a, s}\right)^{n-m}\left[E_{\alpha}\left\{-p_{k}(x)\right\}\right]=\frac{1}{E_{\alpha}\left\{p_{k}(x)\right\}} V_{n-m}^{(\alpha, 0)}(x ; a, k, s),
$$

this gives

$$
\begin{aligned}
& \frac{1}{(n-m) !} \prod_{i=0}^{n-m-1}(s+i a+x D)\left[E_{\alpha}\left\{-p_{k}(x)\right\}\right] \\
= & x^{a(m-n)} \frac{1}{E_{\alpha}\left\{p_{k}(x)\right\}} V_{n-m}^{(\alpha, 0)}(x ; a, k, s) .
\end{aligned}
$$

Use of (3.3) and (3.4) immediately leads to (3.1). 
Proof of (3.2). Writing (1.1) as:

$$
\sum_{n=0}^{\infty} V_{n}^{(\alpha, \beta)}(x ; a, k, s) t^{n}=x^{-\beta} E_{\alpha}\left\{p_{k}(x)\right\} \exp \left(T_{x}^{a, s}\right)\left[x^{\beta} E_{\alpha}\left\{-p_{k}(x)\right\}\right],
$$

where the above equation reduces to

$$
\begin{aligned}
& \sum_{n=0}^{\infty} V_{n}^{(\alpha, \beta)}(x ; a, k, s) t^{n} \\
= & x^{-\beta} E_{\alpha}\left\{p_{k}(x)\right\} x^{\beta}\left(1-a x^{a} t\right)^{-\left(\frac{\beta+s}{a}\right)} E_{\alpha}\left[-p_{k}\left\{x\left(1-a x^{a} t\right)^{-\frac{1}{a}}\right\}\right] \\
= & \left(1-a x^{a} t\right)^{-\left(\frac{\beta+s}{a}\right)} E_{\alpha}\left[p_{k}(x)\right] E_{\alpha}\left[-p_{k}\left\{x\left(1-a x^{a} t\right)^{-\frac{1}{a}}\right\}\right] \\
= & \left(1-a x^{a} t\right)^{-\left(\frac{\gamma+s}{a}\right)} \sum_{m=0}^{\infty}\left(\frac{\beta-\gamma}{a}\right)_{m} \frac{\left(a x^{a} t\right)^{m}}{m !} E_{\alpha}\left[p_{k}(x)\right] \\
& \times E_{\alpha}\left[-p_{k}\left\{x\left(1-a x^{a} t\right)^{-\frac{1}{a}}\right\}\right],
\end{aligned}
$$

on applying equation (1.9), we arrived at

$$
\begin{aligned}
& \sum_{n=0}^{\infty} V_{n}^{(\alpha, \beta)}(x ; a, k, s) t^{n} \\
= & \sum_{m=0}^{\infty}\left(\frac{\beta-\gamma}{a}\right)_{m} \frac{\left(a x^{a} t\right)^{m}}{m !} x^{-\gamma} E_{\alpha}\left\{p_{k}(x)\right\} \exp \left(T_{x}^{a, s}\right)\left[x^{\gamma} E_{\alpha}\left\{-p_{k}(x)\right\}\right] \\
= & \sum_{n=0}^{\infty} \sum_{m=0}^{n}\left(\frac{\beta-\gamma}{a}\right)_{m} \frac{\left(a x^{a}\right)^{m} t^{n}}{m !(n-m) !} x^{-\gamma} E_{\alpha}\left\{p_{k}(x)\right\}\left(T_{x}^{a, s}\right)^{n-m}\left[x^{\gamma} E_{\alpha}\left\{-p_{k}(x)\right\}\right] \\
= & \sum_{m=0}^{\infty} \sum_{n=0}^{\infty}\left(\frac{\beta-\gamma}{a}\right)_{m} \frac{\left(a x^{a}\right)^{m} t^{n+m}}{m ! n !} x^{-\gamma} E_{\alpha}\left\{p_{k}(x)\right\}\left(T_{x}^{a, s}\right)^{n}\left[x^{\gamma} E_{\alpha}\left\{-p_{k}(x)\right\}\right] .
\end{aligned}
$$

Now equating the coefficients of $t^{n}$, we get

$$
\begin{aligned}
V_{n}^{(\alpha, \beta)}(x ; a, k, s)= & \sum_{m=0}^{n} \frac{1}{m !}\left(a x^{a}\right)^{m}\left(\frac{\beta-\gamma}{a}\right)_{m} \frac{x^{-\gamma}}{(n-m) !} \\
& \times E_{\alpha}\left\{p_{k}(x)\right\}\left(T_{x}^{a, s}\right)^{n-m}\left[x^{\gamma} E_{\alpha}\left\{-p_{k}(x)\right\}\right]
\end{aligned}
$$

and use of (1.1) which follows (3.2).

Note: Finite summation formula (3.1) is a particular case of (3.2) for $\gamma=0$ but proof of (3.1) is quite different.

\section{Special cases}

The several interesting special cases of $V_{n}^{(\alpha, \beta)}(x ; a, k, s)$ are as follows:

$$
V_{n}^{(1, \alpha)}(x ; a, k, s)=V_{n}^{(\alpha)}(x ; a, k, s) .
$$


For $p_{k}(x)=p_{1}(x)=x$, we get

$$
\begin{aligned}
& V_{n}^{(1, \alpha+1)}(x ; 1,1,0)=x^{n} L_{n}^{(\alpha)}(x)=x^{n} L_{n}^{1, \alpha}(x) \\
&=x^{n} Z_{n}^{\alpha}(x ; 1)=x^{n} Y_{n}^{\alpha}(x ; 1), \\
& V_{0}^{(0, \beta)}(x ; a, 1, s)=\frac{1}{1-x^{2}} \\
& V_{1}^{(0, \beta)}(x ; a, 1, s)=\frac{x^{a}}{1-x^{2}}\left(s+\beta-\frac{x}{1+x}\right), \\
&\left.V_{0}^{\left(\frac{1}{2}, \beta\right)}(x ; a, 1, s)=\exp \left(2 x^{2}\right)\left(1-(\operatorname{erf}(x))^{2}\right)\right)
\end{aligned}
$$

$$
\begin{aligned}
& V_{1}^{\left(\frac{1}{2}, \beta\right)}(x ; a, 1, s) \\
= & x^{a} \exp \left(x^{2}\right)(1+(\operatorname{erf}(x)))\left[\left(s+\beta+2 x^{2}\right) \exp \left(x^{2}\right)(1-(\operatorname{erf}(x)))-\frac{2 x}{\sqrt{\pi}}\right],
\end{aligned}
$$

$$
\begin{gathered}
V_{0}^{(1, \beta)}(x ; a, 1, s)=1, \\
V_{1}^{(1, \beta)}(x ; a, 1, s)=(s+\beta-x) x^{a}, \\
V_{0}^{(2, \beta)}(x ; a, 1, s)=\cosh \sqrt{x} \cosh \sqrt{-x} \\
V_{1}^{(2, \beta)}(x ; a, 1, s) \\
=x^{a} \cosh (\sqrt{x})\left[(s+\beta) \cosh (\sqrt{-x})+\frac{\sqrt{-x}}{2} \sinh \sqrt{-x}\right],
\end{gathered}
$$

taking $p_{k}(x)=p_{2}(x)=x^{2}$

$$
V_{n}^{(1,0)}(x ;-1,2,0)=\frac{(-x)^{n}}{n !} H_{n}(x) .
$$

\section{A MAPLE implementation}

Zeilberger [10] gave MAPLE programme for proving the hypergeometric identities. In the light of his work, we also give MAPLE program for the symbolic representations of sequence of functions (1.1). 


\section{The MAPLE program Listing:}

$>\mathrm{A}:=\operatorname{proc}(\mathrm{x}, \mathrm{y})$ options operator, arrow; $\mathrm{x}^{*}(\operatorname{diff}(\mathrm{y}, \mathrm{x}))$ end proc;

$$
A:(x, y) \rightarrow x\left(\frac{\partial}{\partial u} y\right) .
$$

$>\mathrm{B}:=\operatorname{proc}(\mathrm{s}, \mathrm{y})$ options operator, arrow; $\mathrm{s}^{*} \mathrm{y}$ end proc;

$$
B:(s, y) \rightarrow s .
$$

$>\mathrm{C}:=\operatorname{proc}(\mathrm{s}, \mathrm{x}, \mathrm{y})$ options operator, arrow;s, $\mathrm{x}, \mathrm{B}(\mathrm{s}, \mathrm{y})+\mathrm{A}(\mathrm{x}, \mathrm{y})$ end proc;

$$
C:(s, x, y) \rightarrow s, x, B(s, y)+A(x, y) .
$$

$>\mathrm{K}:=\operatorname{proc}(\mathrm{a}, \mathrm{b}, \mathrm{c})$ options operator, arrow; c end proc;

$$
K:(a, b, c) \rightarrow c .
$$

$>\mathrm{E}:=\operatorname{proc}($ alpha, $\mathrm{x})$ options operator, arrow; $\operatorname{sum}\left(\mathrm{x}^{\wedge} \mathrm{r} / \mathrm{GAMMA}(\right.$ alpha*r+1$)$, $\mathrm{r}=0 \ldots$ infinity) end proc;

$$
E:=(\alpha, x) \rightarrow \sum_{r=0}^{\infty} \frac{x^{r}}{\Gamma(\alpha r+1)} .
$$

$>\mathrm{F}:=\operatorname{proc}($ alpha, beta, $\mathrm{n}, \mathrm{a}, \mathrm{k}, \mathrm{s}, \mathrm{x})$ options operator, arrow; $\mathrm{x}^{\wedge}\left(\mathrm{a}^{*} \mathrm{n}\right) * \mathrm{~K}$ $\left((\mathrm{C} @ \mathrm{n})\left(\mathrm{s}, \mathrm{x}, \mathrm{x}^{\wedge}\right.\right.$ beta* $^{*} \mathrm{E}\left(\right.$ alpha, $\left.\left.\left.-\mathrm{x}^{\wedge} \mathrm{k}\right)\right)\right) /$ factorial(n) end proc;

$$
F:=(\alpha, \beta, n, a, k, s, x) \rightarrow \frac{x^{a n} K\left(C^{(n)}\left(s, x, x^{\beta} E\left(\alpha,-x^{k}\right)\right)\right)}{n !} .
$$

$>\mathrm{V}:=\operatorname{proc}($ alpha, beta, $\mathrm{n}, \mathrm{a}, \mathrm{k}, \mathrm{s}, \mathrm{x})$ options operator, arrow; $\mathrm{x}^{\wedge}($-beta)* $\mathrm{E}$ $\left(\right.$ alpha, $\left.\mathrm{x}^{\wedge} \mathrm{k}\right) * \mathrm{~F}$ (alpha, beta, $\left.\mathrm{n}, \mathrm{a}, \mathrm{k}, \mathrm{s}, \mathrm{x}\right)$ end proc;

$$
V:=(\alpha, \beta, n, a, k, s, x) \rightarrow x^{-\beta} E\left(\alpha, x^{k}\right) F(\alpha, \beta, n, a, k, s, x) .
$$

\section{Instructions:}

To run the MAPLE program, start new MAPLE windows in 'WORKSHEET MODE' with default 'TYPESETTING RULES'. Type the above MAPLE programme in '1-D Math input'. Type ' $\mathrm{V}$ (alpha, beta, $\mathrm{n}, \mathrm{a}, \mathrm{k}, \mathrm{s}, \mathrm{x}$ );' for $\mathrm{n}$ $=0,1,2, \ldots$ and press 'Enter' then MAPLE gives the symbolic expression for $V(\alpha, \beta, n, a, k, s, x)$. We cannot evaluate directly $V(\alpha, \beta, n, a, k, s, x)$ without entering the integer value of $n$ due to $\left(T_{x}^{a, s}\right)^{n}$, where $T_{x}^{a, s} \equiv x^{a}(s+x D)$ and $D \equiv \frac{d}{d x}$ are operators.

\section{Graphs:}

Some graphs of new sequence of functions (1.1) are listed below: 


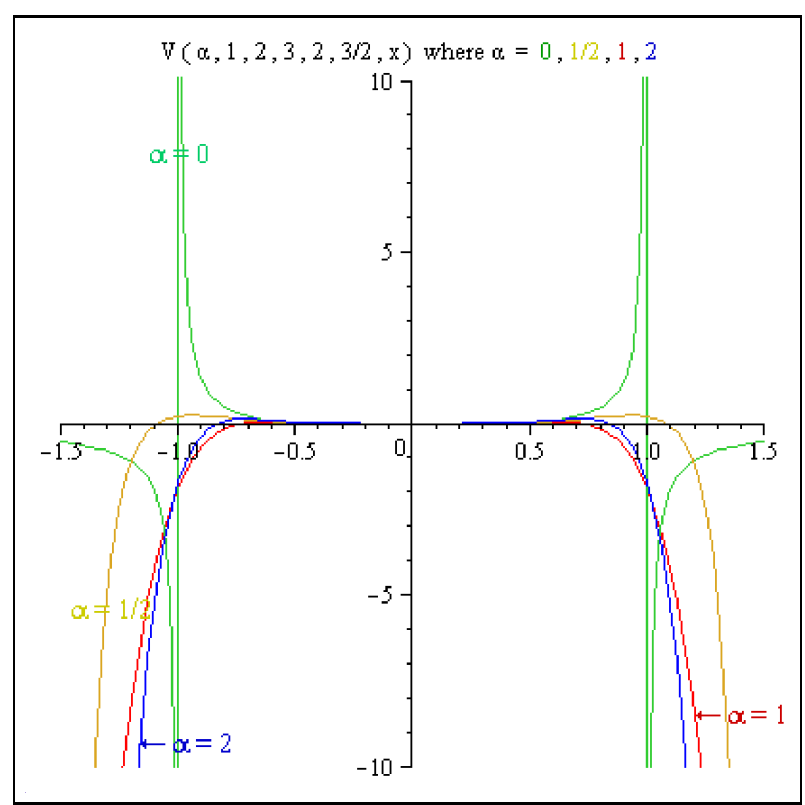

FIGURE 1

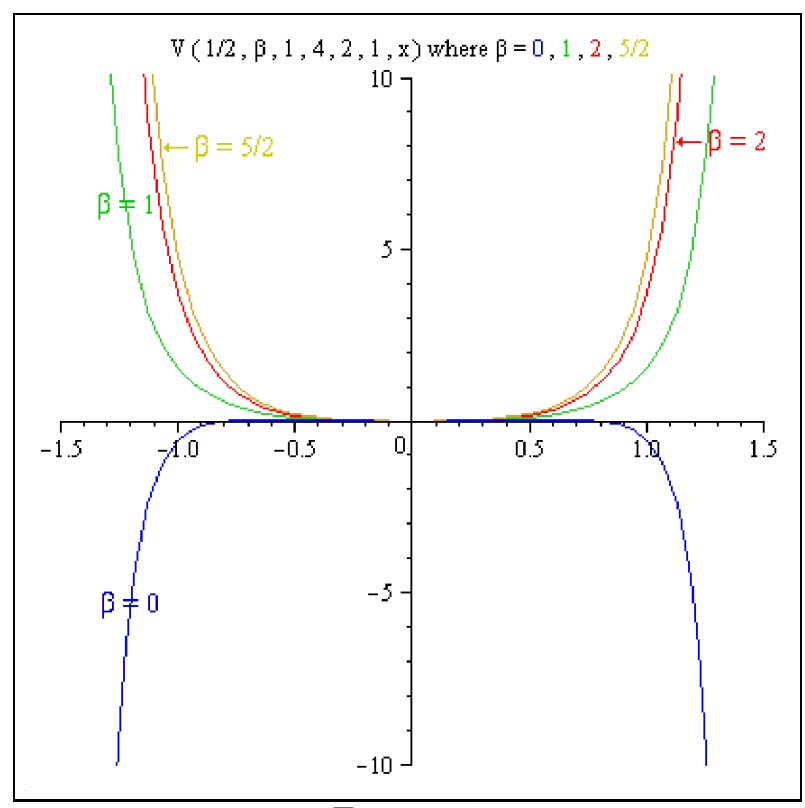

FIGURE 2 


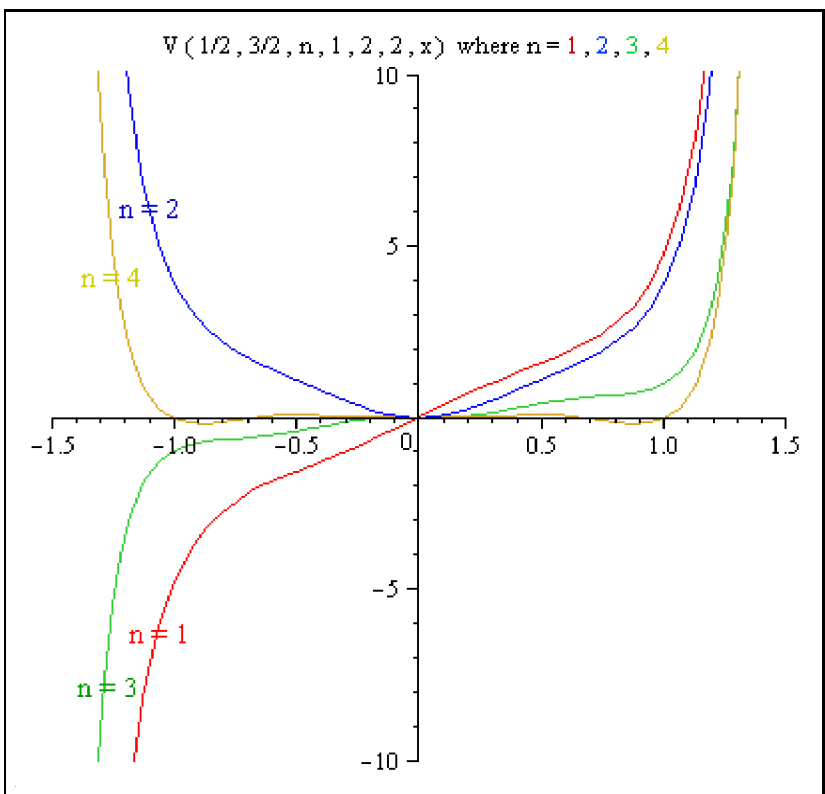

FIGURE 3

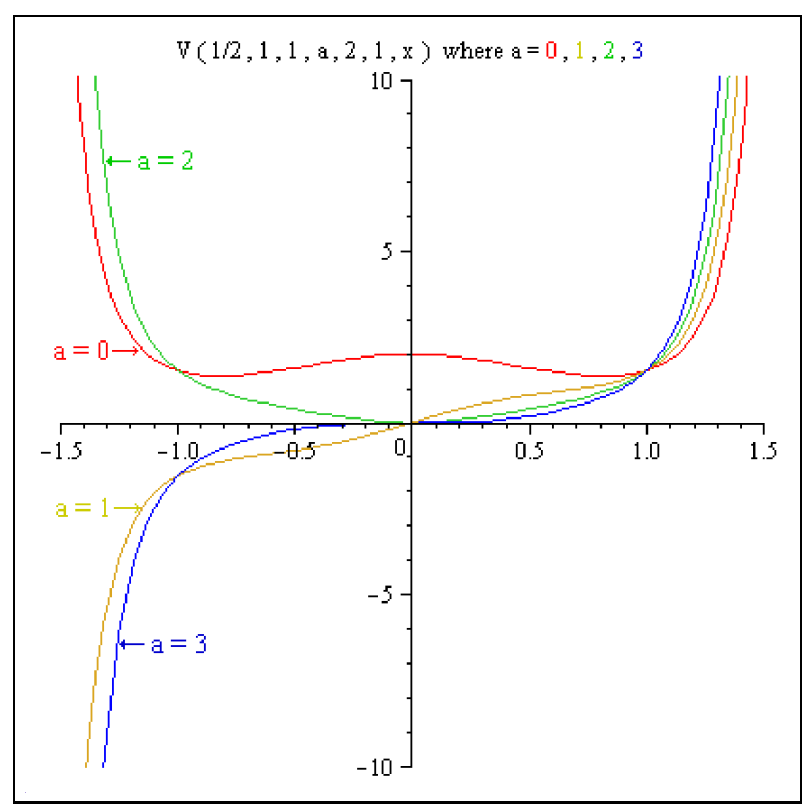

FIGURE 4 


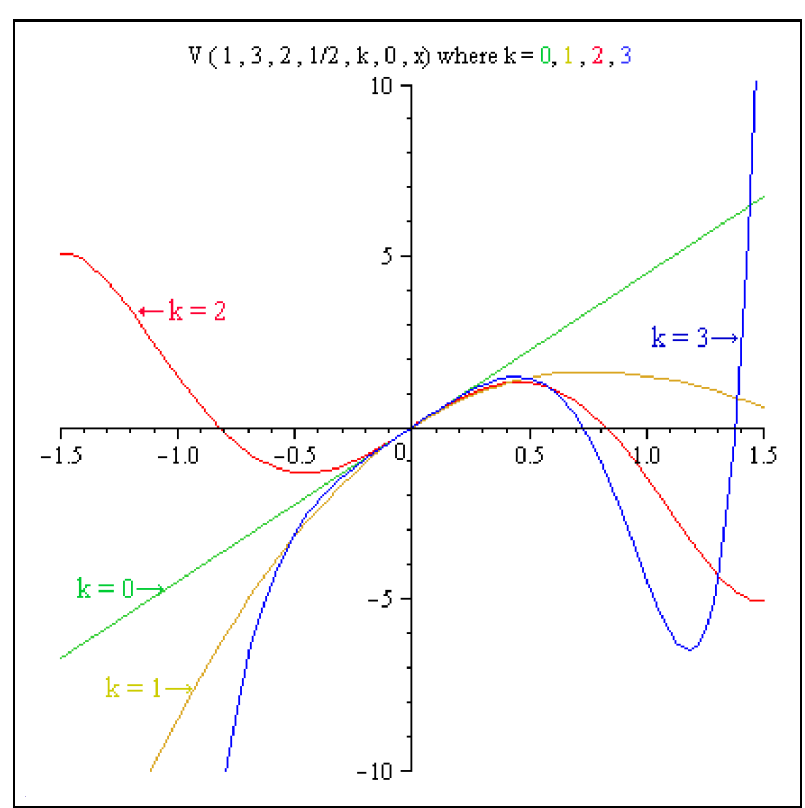

FigURE 5

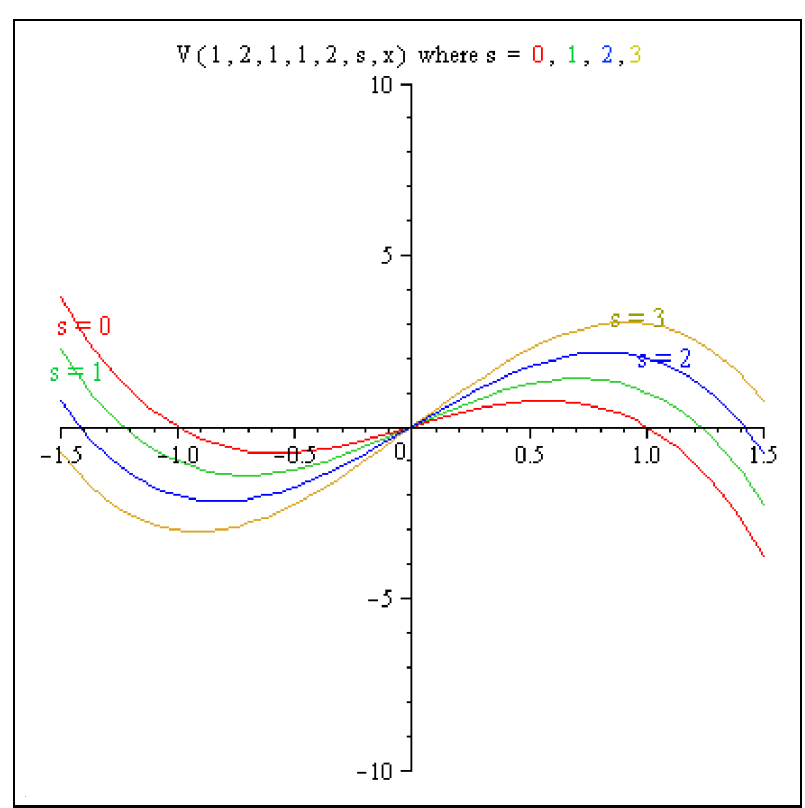

FIGURE 6 
Acknowledgement. First author is thankful to Council of Scientific and Industrial Research, New Delhi for awarding Senior Research Fellowship (Award No.: File no. 09 /1007/(0001)/2010/ EMR-I dated 13/03/2010).

\section{References}

[1] E. J. Berg, Heaviside's Operational Calculus, New York, McGraw-Hill, 1929.

[2] M. N. Berberan-Santos, Properties of the Mittag-Leffler relaxation function, J. Math. Chem. 38 (2005), no. 4, 629-635.

[3] H. B. Mittal, Bilinear, and Bilateral generating relations, Amer. J. Math. 99 (1977), no. $1,23-45$.

[4] K. R. Patil and N. K. Thakare, Operational formulas for a function defined by a generalized Rodrigues formula-II, Sci. J. Shivaji Univ. 15 (1975), 1-10.

[5] T. R. Prabhakar and R. Suman, Some results on the polynomials, Rocky Mountain J. Math. 8 (1978), no. 4, 751-754.

[6] E. D. Rainville, Special Functions, The Macmillan Company, New York, 1960.

[7] A. N. Srivastava and S. N. Singh, Some generating relations connected with a function defined by a generalized Rodrigues formula, Indian J. Pure Appl. Math. 10 (1979), no. $10,1312-1317$.

[8] H. M. Srivastava, Some biorthogonal polynomials suggested by the Laguerre polynomials, Pacific J. Math. 98 (1982), no. 1, 235-250.

[9] H. M. Srivastava and H. L. Manocha, A Treatise on Generating Functions, Ellis Horwood Limited, Chichester, 1984.

[10] D. Zeilberger, A MAPLE program for proving hypergeometric Identities, SIGSAM Bulletin 25 (1991), no. 3, 4-13.

IBRAHim A. SALEHBHAI

Department of Applied Mathematics \& Humanities

S.V. National Institute of Technology

SuRAT-395 007, InDia

E-mail address: ibrahimmaths@gmail.com

Jyotindra C. Prajapati

Department of Mathematical Sciences

Faculty of Applied Sciences

Charotar University of Science and Technology

Changa, Anand-388421, India

E-mail address: jyotindra18@rediffmail.com

AJAY K. SHUKLa

Department of Applied Mathematics \& Humanities

S.V. National Institute of Technology

SuRAT-395 007, India

E-mail address: ajayshukla2@rediffmail.com 